\title{
TRATAMENTOS PARA ACELERAR A GERMINAÇÃO E REDUZIR A DETERIORAÇÃO DAS SEMENTES DE Ormosia nitida VOG. ${ }^{1}$
}

\author{
José Carlos Lopes², Paulo César Dias³ e Célia Maria Peixoto de Macedo³
}

\begin{abstract}
RESUMO - O trabalho foi conduzido no Laboratório de Sementes do Centro de Ciências Agrárias da Universidade Federal do Espírito Santo, objetivando avaliar tratamentos para acelerar a germinação e reduzir a deterioração de sementes de Ormosia nitida. Foram conduzidos dois experimentos em delineamento inteiramente casualizado, com quatro repetições. No primeiro estudo, feito em papel substrato, os tratamentos foram sementes intactas, escarificação mecânica e escarificação química com $\mathrm{H}_{2} \mathrm{SO}_{4}$ durante $10 \mathrm{~min}$. No segundo estudo feito em placas de Petri, os tratamentos utilizados foram sementes intactas (controle); escarificação mecânica; escarificação mecânica + pré-embebição por 24 horas; $\mathrm{H}_{2} \mathrm{O}_{2}$ no substrato; e escarificação química com $\mathrm{H}_{2} \mathrm{SO}_{4}$ durante 1 , $5,10,15,20,25$ e $30 \mathrm{~min}$. Foram avaliados o vigor, através do índice de velocidade de germinação, e a germinação, através da porcentagem de plântulas normais. A todos os tratamentos pré-germinativos foram apresentados respostas positivas na porcentagem e velocidade de germinação em relação ao controle. A escarificação mecânica e o $\mathrm{H}_{2} \mathrm{O}_{2}$ no substrato proporcionaram aumento significativo na velocidade e porcentagem de germinação das sementes, com redução da deterioração.
\end{abstract}

Palavras-chave: Ormosia nitida, capacidade germinativa, vigor e dormência.

\section{TREATMENTS TO ACCELERATE GERMINATION AND REDUCE DETERIORATION OF Ormosia nitida SEEDS}

\begin{abstract}
The experiment was carried out at the Seed Laboratory of the Agronomy Center of the Federal University of Espirito Santo, in order to attain acceleration of germination and reduce seed deterioration in Ormosia nitida Vog.. A completely randomized experimental design with four replications was used. In experiment I, with paper substratum, the treatments were intact seeds (control); mechanical scarification and sulfuric acid soaking for 10 minutes. In experiment II, in Petri dishes, the treatments were intacts seeds (control); mechanical scarification; mechanical scarification + soaking in water at $35^{\circ} \mathrm{C}$ for 24 hours; imbibition of germination substratum with $\mathrm{H}_{2} \mathrm{O}_{2}$; sulphuric acid soaking for 1; 5; 10; 15;20; 25 and 30 minutes. The results showed higher synchronization and speed germination in treatments with mechanical scarification, sulphuric acid for 10 minutes and $\mathrm{H}_{2} \mathrm{O}_{2}$ in the substratum.
\end{abstract}

Keywords: Ormosia nitida, germinative capacity, vigor and dormancy.

\section{INTRODUÇÃO}

A espécie em estudo Ormosia nitida Vog., devido à beleza de suas flores, rápido crescimento e porte, apresenta grande potencial para utilização em projetos paisagísticos e na ornamentação de ruas e praças (CORRÊA, 1978).

A germinação tem início com a absorção de água pela semente e termina com o alongamento do eixo embrionário, em que a protrusão do embrião através do tegumento é o ponto crucial que identifica esse

\footnotetext{
${ }^{1}$ Recebido em $1^{\circ} .06 .2004$ e aceito para publicação em 10.11.2005.

${ }^{2}$ Departamento de Fitotecnia do Centro de Ciências Agrárias da Universidade Federal do Espírito Santo (CCAUFES), Alto Universitário, Cx. Postal 16, 29500-000 Alegre-ES. E-mail: <jclopes@cca.ufes.br>.

${ }^{3}$ Santo Antonio, Zona Rural, Piaçú, Cx. Postal 13, 29386-000 Muniz Freire-ES. E-mail: <diaspc1 @ yahoo.com.br>.

${ }^{4}$ Programa de Pós-Graduação em Produção Vegetal/ CCA-UFES. E-mail: <celiampm@yahoo.com.br>.
} 
processo (BEWLEY e BLACK, 1994). Entretanto, para que ela ocorra, é necessário, além do substrato, que haja disponibilidade de água em níveis ideais, de acordo com o tipo de semente; composição de gases e temperatura adequada, afora luz, que é exigida para certas espécies; e que a semente esteja viável, isto é, que o embrião esteja vivo e capaz de germinar e que esteja no estado quiescente, de tal forma que, quando submetida a condições consideradas ideais, ela germine (MAYER e POLJAKOFF-MAYBER, 1989).

O tegumento, além de restringir ou regular a entrada de água na semente, agindo como uma barreira mecânica à difusão, desempenha funções vitais necessárias para o seu bom desenvolvimento, manutenção, viabilidade e perpetuação da espécie. Existem sementes que, devido a características físicas e químicas do tegumento, apresentam estrutura e consistência compactas e impermeáveis à água e gases e inibições mecânica e química da germinação. Tal fenômeno constitui fator limitante à propagação da espécie, ocorrendo, principalmente, em leguminosas e outras famílias que possuem sementes com tegumento duro e impermeável (MAYER e POLJAKOFF MAYBER, 1989; BEWLEY e BLACK, 1994). Nesse tipo de dormência, a remoção do tegumento ou a escarificação química ou mecânica aumenta a permeabilidade à água, a sensibilidade à luz e à temperatura, permeabilidade a gases, remoção de inibidores ou de promotores da germinação, influenciando o metabolismo e, conseqüentemente, a dormência das sementes, como em Senna macranthera (SANTARÉMeAQUILA, 1995; ESCHIAPATI-FERREIRA e PEREZ, 1997); Caesalpinea ferrea, Cassia grandis e Samanea saman (LOPES et al., 1998); Cassia excelsa (JELLER e PEREZ, 1999); e Mimosa caesalpiniaefolia (BRUNO et al., 2001).

Em sementes de Ormosia arborea, Lorenzi (1998) recomendou a escarificação mecânica antes da semeadura para aumentar a germinação e, no caso das sementes de Ormosia nitida, embora não haja informações, elas apresentam vários obstáculos à germinação, pelo fato de serem recobertas por um tegumento duro, que restringe a passagem de água e pode estar relacionada à impermeabilidade do tegumento (BEWLEY e BLACK, 1994). Assim, neste estudo objetivou-se avaliar diferentes tratamentos para acelerar a germinação e reduzir a deterioração das sementes de Ormosia nitida Vog.

\section{MATERIAL E MÉTODOS}

O trabalho foi conduzido no Laboratório de Sementes do Centro de Ciências Agrárias da Universidade Federal do Espírito Santo, em Alegre, ES (CCA-UFES). Foram utilizadas sementes recém-colhidas (junho de 2001) de Ormosia nitida Vog. provenientes da Floresta Rio Doce, em Linhares, ES, e da Serra do Caparaó, em Ibitirama, ES.

Para o estudo da embebição foram utilizadas duas repetições de $5 \mathrm{~g}$ de sementes, colocadas em béquer com $15 \mathrm{~mL}$ de água destilada, à temperatura constante de $30^{\circ} \mathrm{C}$. Periodicamente, as sementes eram removidas dos béqueres e secadas em papel absorvente, sendo as pesagens feitas a cada hora num período de $8 \mathrm{~h}$ e após 24,48 e $72 \mathrm{~h}$. A embebição foi considerada como o aumento de peso em relação ao peso inicial.

O estudo da germinação foi feito utilizando-se sementes tratadas por imersão durante dois minutos em hipoclorito de sódio $5 \%$, seguido da imersão em benlate (BENOMYL 500), na concentração de $750 \mathrm{mg} \mathrm{L}^{-1}$.

Foram realizados dois experimentos. No primeiro, conduzido em rolos de papel-toalha como substrato, umedecido com água destilada equivalente a 2,5 vezes o seu peso, os tratamentos utilizados foram: sementes intactas (controle), escarificação mecânica com lixa de madeira $\mathrm{n}^{\circ} 60$ e escarificação química com $\mathrm{H}_{2} \mathrm{SO}_{4}$, durante $10 \mathrm{~min}$. No segundo, conduzido em placas de Petri previamente esterilizadas, forradas com papelfiltro, umedecido com $15 \mathrm{~mL}$ de água destilada, os tratamentos empregados foram: sementes intactas (controle); escarificação mecânica; escarificação mecânica + pré-embebição por $24 \mathrm{~h} ; \mathrm{H}_{2} \mathrm{O}_{2}$ no substrato; e escarificação química com $\mathrm{H}_{2} \mathrm{SO}_{4}$, durante $1,5,10,15$, 20,25 e $30 \mathrm{~min}$.

A germinação foi avaliada através da porcentagem de plântulas normais (BRASIL, 1992) e a velocidade de germinação, através de um índice determinado pela fórmula de Maguire (1962). A porcentagem de deterioração foi calculada em conjunto com o teste de germinação, considerando as sementes mortas no final do teste. Com os dados de germinação diária foram calculados, ainda, o tempo médio e a freqüência relativa de germinação (LABOURIAU e VALADARES, 1976).

O delineamento experimental utilizado foi o inteiramente casualizado com quatro subamostras de 20 sementes no experimento I e quatro subamostras 
de 25 sementes no experimento II. As porcentagens de germinação e deterioração foram transformadas para arco-seno $\sqrt{X / 100}$ e os valores do índice de velocidade de germinação, transformados em $\sqrt{X+0.5}$ para fins de análise estatística. Os dados para interpretação foram apresentados com as médias dos dados originais. As médias de germinação, deterioração e índice de velocidade de germinação foram comparados através do teste de Tukey $(\alpha=0,05)$.

\section{RESULTADOS E DISCUSSÃO}

Na Figura 1, apresenta-se a embebição das sementes de Ormosia nitida intactas e após o tratamento com escarificações mecânica e química. De acordo com os resultados, verificou-se que as sementes intactas e as tratadas com escarificação química não tiveram aumento no conteúdo de matéria fresca, mantendo seus valores bastante próximos, enquanto as sementes que foram tratadas com escarificação mecânica exibiram aumento progressivo e paulatino na matéria fresca, caracterizando maior dureza e impermeabilidade do tegumento dessas sementes à água (BEWLEY e BLACK, 1994).

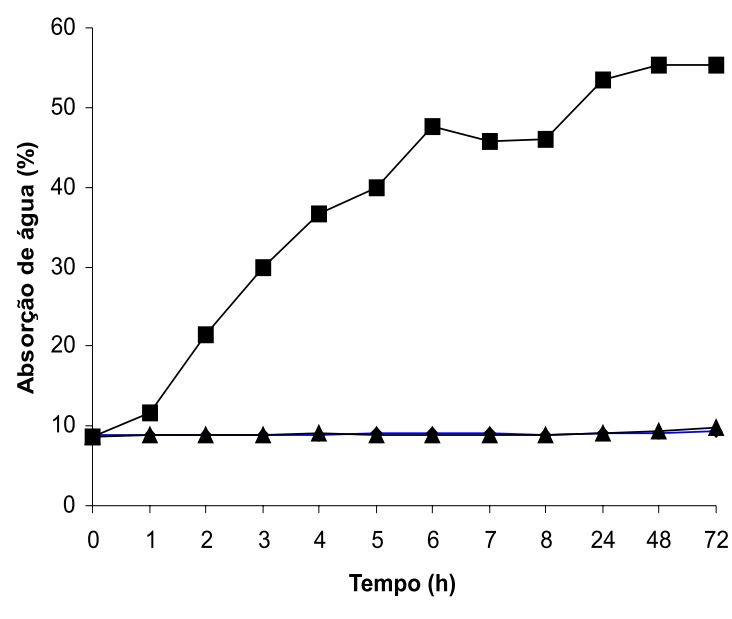

Intacta -Escarificação Mecânica $\longrightarrow$ -

Figura 1 - Teor de água em sementes de Ormosia nitida Vog., após a embebição em água destilada. CCA/UFES, Alegre, ES, 2004.

Figure 1 - Water content of Ormosia nitida Vog. seeds, after imbibition in distillated water. CCA/UFES, Alegre, ES, 2004.
Na Tabela 1, mostram-se os resultados de germinação das sementes obtidos no experimento I. Verifica-se, nessa tabela, que os tratamentos feitos com escarificações mecânica e química com $\mathrm{H}_{2} \mathrm{SO}_{4}$, durante $10 \mathrm{~min}$, determinaram aumento acentuado na velocidade e porcentagem de germinação das sementes de Ormosia nitida, em relação ao controle. Nesses tratamentos houve pequena incidência de sementes deterioradas, sendo que o controle apresentou alta porcentagem de sementes duras, com maior tempo médio de germinação $(15,41)$, em relação à escarificação mecânica $(6,04)$ e à química $(6,27)$ (Figura 2), em que se verifica que os polígonos da distribuição da freqüência relativa mostraram unimodalidade nos tratamentos realizados com escarificação, enquanto no controle se observou polimodalidade.

No experimento II (Tabela 2), de acordo com os resultados, todos os tratamentos pré-germinativos apresentaram respostas positivas na porcentagem e velocidade de germinação em relação ao controle. Os valores médios da germinação e do índice de velocidade de germinação evidenciaram que os tratamentos feitos com escarificação mecânica e com $\mathrm{H}_{2} \mathrm{O}_{2}$ no substrato proporcionaram aumento significativo na germinação ( 100 e $93 \%$, respectivamente) e na velocidade de germinação (3,23 e 3,49, respectivamente), em relação às sementes não-tratadas (controle). Bianchetti e Ramos (1982), trabalhando com Peltophorum dubium, obtiveram bons resultados com vários tratamentos com escarificação mecânica. Em sementes de Bauhinia ungulata, Alves et al. (2000) constataram que a escarificação mecânica foi o tratamento mais eficiente para a superação da dormência.

A escarificação mecânica seguida de pré-embebição e os tratamentos feitos com imersão em ácido sulfúrico por $1,5,10,15,20,25$ e $30 \mathrm{~min}$, embora tenham revelado porcentagens de germinação estatisticamente similares às dos tratamentos com escarificação mecânica e aplicação de $\mathrm{H}_{2} \mathrm{O}_{2}$ no substrato, esses valores, contudo, não diferiram significativamente do controle. Entretanto, verificou-se aumento acentuado na porcentagem de sementes deterioradas, evidenciando que esses tratamentos, sempre que possível, devem ser evitados, principalmente pelo grau de periculosidade que oferecem. Esses resultados indicam que a permanência das sementes por períodos mais prolongados no ácido, embora supere o fenômeno da dormência, aumenta significativamente

R. Árvore, Viçosa-MG, v.30, n.2, p.171-177, 2006 
a porcentagem de sementes deterioradas e de plântulas anormais, culminando com alta incidência de fungos e bactérias. Entretanto, a velocidade de germinação obtida em todos os tratamentos foi significativamente maior do que a do controle. Bruno et al. (2001) também obtiveram aumento na velocidade de germinação em sementes de Mimosa caesalpineaefolia tratadas com $\mathrm{H}_{2} \mathrm{SO}_{4}$, durante sete, 10 e $13 \mathrm{~min}$.

No experimento II, similarmente aos resultados obtidos no experimento I (Figura 2), os polígonos da distribuição da freqüência relativa de Ormosia nitida (Figuras 3 e 4) evidenciaram a polimodalidade para os tratamentos feitos com $\mathrm{H}_{2} \mathrm{O}_{2}$ no substrato e imersão das sementes em ácido sulfúrico, em que os polígonos de freqüência relativa apresentaram deslocamento do tempo médio para a esquerda, indicando aumento na velocidade do processo germinativo, enquanto na escarificação mecânica se verificou unimodalidade. Bianchetti e Ramos (1982), trabalhando com Peltophorum dubium, obtiveram bons resultados com vários tratamentos com escarificações mecânica e escarificação química com ácido sulfúrico, enquanto Eschiapatia-Ferreira e Perez (1997) e Bruno et al. (2001), trabalhando, respectivamente, com Senna macranthera e Mimosa caesalpiniaefolia, conseguiram os melhores resultados na germinação após realizarem escarificação química da semente.

Tabela 1 - Germinação (\%), índice de velocidade de germinação (IVG), deterioração (\%), sementes duras (\%) de sementes de Ormosia nitida Vog., com diferentes tratamentos. CCA-UFES, Alegre, ES, 2004

Table 1 - Standard germination (\%), speed germination index (IVG), deterioration (\%), hard seeds (\%) of Ormosia nitida Vog. seeds, after different treatments. CCA-UFES, Alegre, ES, 2004

\begin{tabular}{|c|c|c|c|c|}
\hline Tratamentos & Germinação (\%) & IVG & Deterioração (\%) & Sementes duras (\%) \\
\hline Controle & $40,00 \quad b$ & 0,5429 & $1,25 \mathrm{a}$ & 58,75 a \\
\hline Escarificação mecânica & 93,75 a & 3,3662 a & $5,00 \mathrm{a}$ & $1,25 \quad b$ \\
\hline $\mathrm{H}_{2} \mathrm{SO}_{4} 10^{\prime}$ & 96,25 a & 3,2386 a & $1,25 \mathrm{a}$ & 2,50 \\
\hline
\end{tabular}

*Médias seguidas pela mesma letra na coluna não diferem significativamente entre si, pelo teste de Tukey a 5\% de probabilidade.

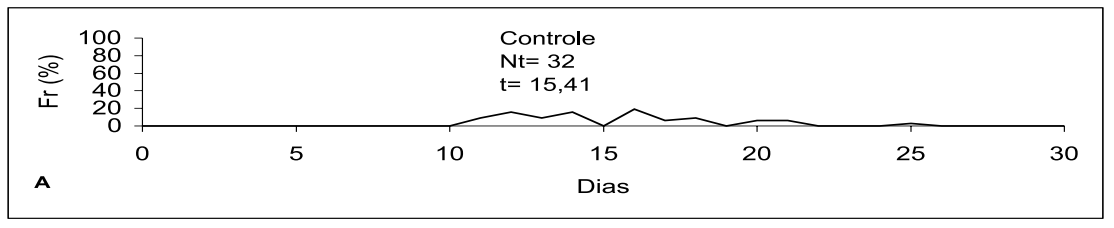

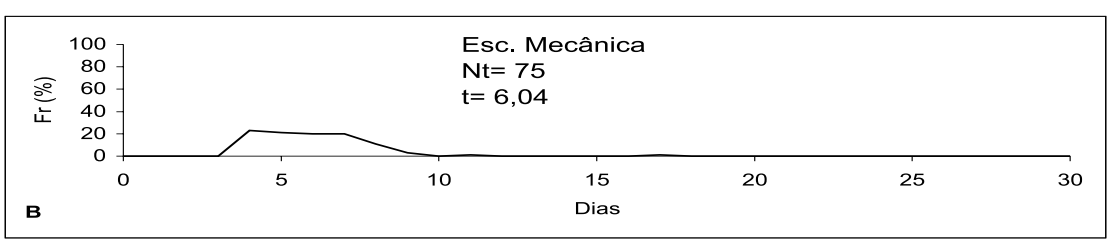

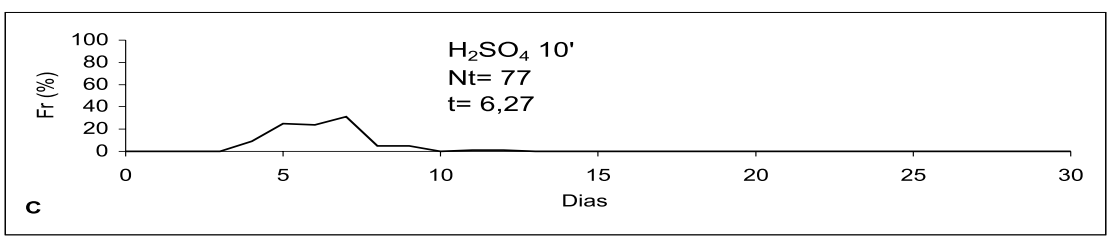

Figura 2 - Frequiência relativa (Fr) da germinação de sementes de Ormosia nitida após diferentes tratamentos pré-germinativos. Laboratório de Sementes, CCA-UFES, Alegre, ES, 2004 ( Nt = número total de sementes germinadas e $\mathrm{t}=$ tempo médio de germinação).

Figure 2 - Relative frequency $(F r)$ of germination of Ormosia nitida Vog. seeds, after different treatments. CCA-UFES, Alegre, ES, 2004.

R. Árvore, Viçosa-MG, v.30, n.2, p.171-177, 2006 
Tratamentos para acelerar a germinação e reduzir ...

Tabela 2 - Germinação (\%), Índice de Velocidade de Germinação (IVG), deterioração (\%), sementes duras (\%) de sementes de Ormosia nitida Vog.. Laboratório de Sementes, CAUFES, Alegre, ES, 2004

Table 2 - Standard germination (\%), speed germination index (IVG), deterioration (\%), hard seeds (\%) of Ormosia nitida Vog. seeds, after different treatments. CCA-UFES, Alegre, ES, 2004

\begin{tabular}{lcccc}
\hline Tratamentos & Germinação (\%) & IVG & Deterioração (\%) & \multicolumn{2}{c}{ Sementes duras (\%) } \\
\hline $\mathrm{H}_{2} \mathrm{O}_{2}$ & $93,00 \mathrm{a}$ & $3,49 \mathrm{a}$ & $0 \mathrm{c}$ & $7,00 \mathrm{c}$ \\
Escarificação mecânica & $100,00 \mathrm{a}$ & $3,23 \mathrm{a}$ & $0 \mathrm{c}$ & $0,00 \mathrm{~d}$ \\
Esc. Mec. + pré-embebição & $87,00 \mathrm{ab}$ & $2,63 \mathrm{a}$ & $1 \mathrm{bc}, 00 \mathrm{bc}$ \\
$\mathrm{H}_{2} \mathrm{SO}_{4} / 1$ minuto & $71,00 \mathrm{ab}$ & $2,75 \mathrm{a}$ & $24 \mathrm{a}$ & $5,00 \mathrm{~cd}$ \\
$\mathrm{H}_{2} \mathrm{SO}_{4} / 5$ minutos & $84,00 \mathrm{ab}$ & $3,49 \mathrm{a}$ & $16 \mathrm{ab}$ & $0,00 \mathrm{~d}$ \\
$\mathrm{H}_{2} \mathrm{SO}_{4} / 10$ minutos & $72,00 \mathrm{ab}$ & $3,44 \mathrm{a}$ & $27 \mathrm{a}$ & $1,00 \mathrm{~d}$ \\
$\mathrm{H}_{2} \mathrm{SO}_{4} / 15$ minutos & $80,00 \mathrm{ab}$ & $3,21 \mathrm{a}$ & $6 \mathrm{bc}$ & $14,00 \mathrm{~b}$ \\
$\mathrm{H}_{2} \mathrm{SO}_{4} / 20$ minutos & $85,00 \mathrm{ab}$ & $3,89 \mathrm{a}$ & $8 \mathrm{bc}$ & $7,00 \mathrm{c}$ \\
$\mathrm{H}_{2} \mathrm{SO}_{4} / 25$ minutos & $83,00 \mathrm{ab}$ & $3,74 \mathrm{a}$ & $16 \mathrm{ab}$ & $1,00 \mathrm{~d}$ \\
$\mathrm{H}_{2} \mathrm{SO}_{4} / 30$ minutos & $72,00 \mathrm{ab}$ & $3,04 \mathrm{a}$ & $11 \mathrm{abc}$ & $17,00 \mathrm{~b}$ \\
Controle & $48,00 \mathrm{~b}$ & $1,04 \mathrm{~b}$ & $11 \mathrm{abc}$ & $41,00 \mathrm{a}$ \\
\hline
\end{tabular}

*Médias seguidas da mesma letra na coluna não diferem significativamente entre si, pelo teste de Tukey a 5\% de probabilidade.
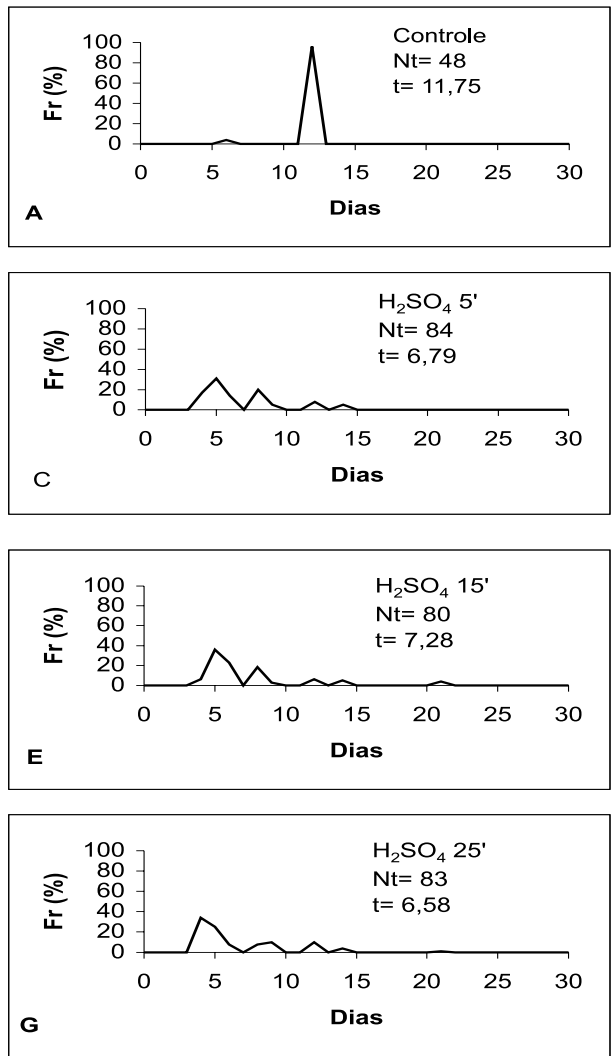
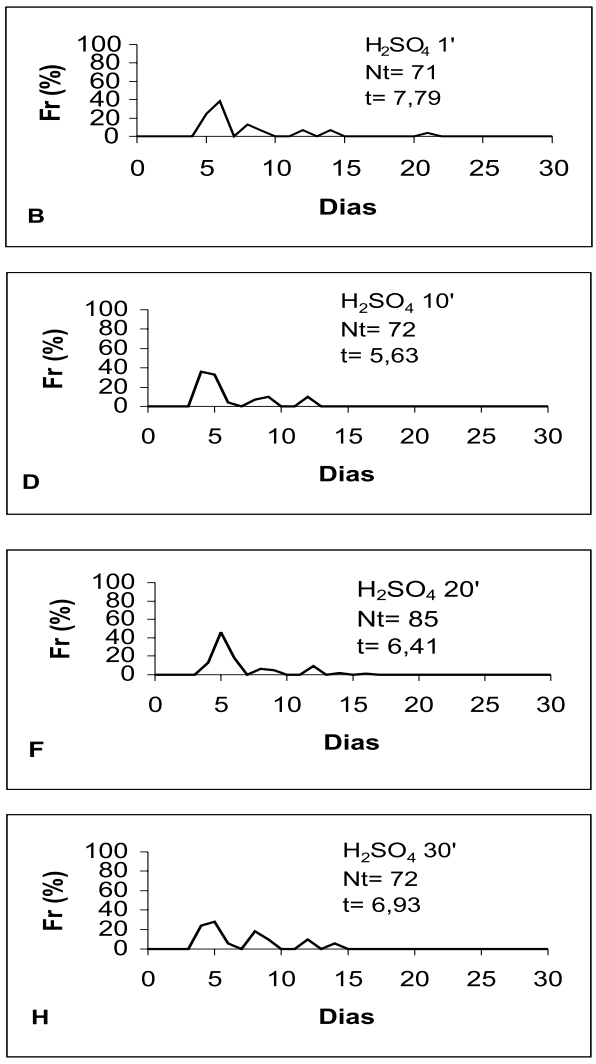

Figura 3 - Freqüência relativa (Fr) da germinação de sementes de Ormosia nitida após vários tempos de exposição ao ácido sulfúrico. Laboratório de Sementes, CCA-UFES, Alegre, ES, 2004 ( Nt = número total de sementes germinadas e $\mathrm{t}=$ tempo médio de germinação).

Figure 3 - Relative frequency $(F r)$ of germination of Ormosia nitida Vog. seeds, after different times of exposure to sulfuric acid. CCA-UFES, Alegre, ES, 2004. 

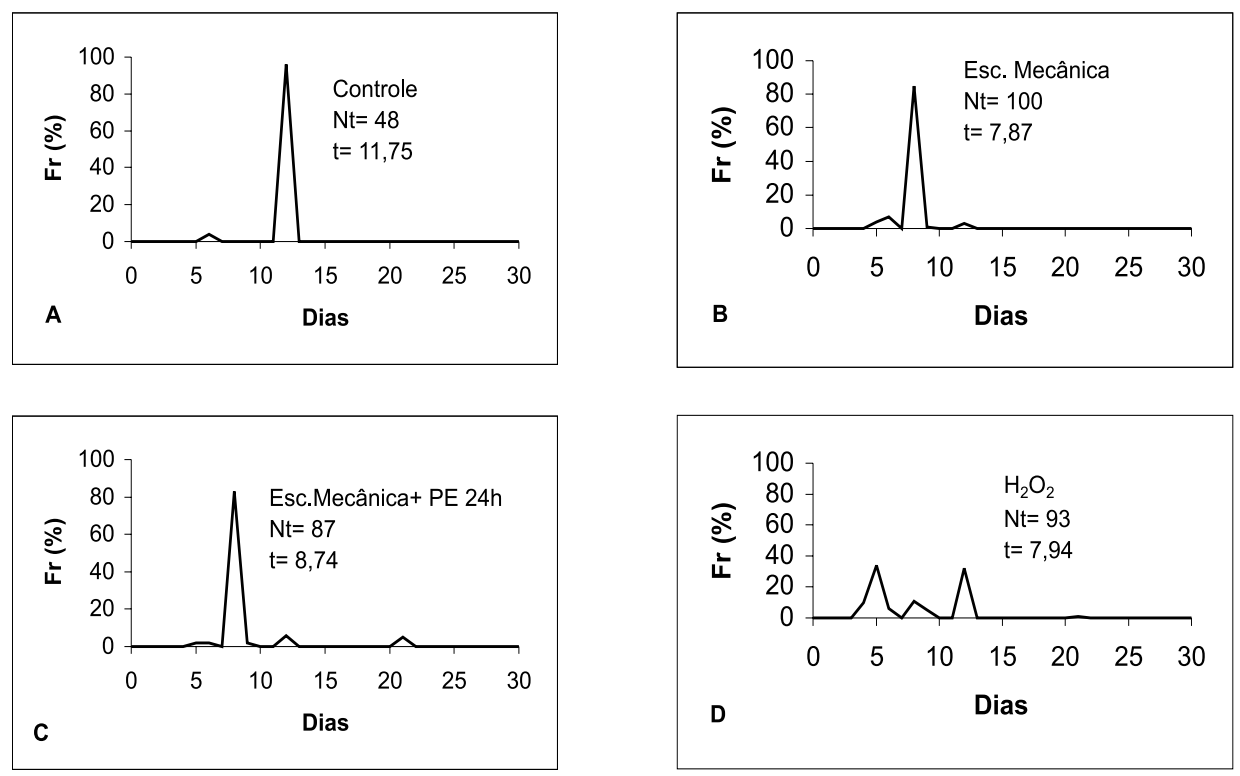

Figura 4 - Freqüência relativa (Fr) da germinação de sementes de Ormosia nitida após diferentes tratamentos pré-germinativos. Laboratório de Sementes, CCA-UFES, Alegre, ES, $2004(\mathrm{Nt}=$ número total de sementes germinadas e $\mathrm{t}=$ tempo médio de germinação).

Figure 4-Relative frequency $(\mathrm{Fr})$ of germination of Ormosia nitida Vog. seeds, after different treatments. CCA-UFES, Alegre, ES, 2004.

\section{CONCLUSÕES}

Nas condições em que foi desenvolvido este trabalho, concluiu-se que:

* As sementes de Ormosia nitida evidenciaram mecanismo de dormência, que é relacionada à impermeabilidade do tegumento.

* Os tratamentos com escarificação mecânica, $\mathrm{H}_{2} \mathrm{O}_{2}$ e ácido sulfúrico reduzem a dormência e aumentam a velocidade e porcentagem de germinação das sementes de Ormosia nítida.

* Tratamentos com escarificação mecânica e $\mathrm{H}_{2} \mathrm{O}_{2}$ diminuem a deterioração das sementes de Ormosia nitida.

\section{AGRADECIMENTOS}

Ao Conselho Nacional de Pesquisa e Desenvolvimento, pela concessão da bolsa de apoio técnico à pesquisa à eng ${ }^{\mathrm{a}}$. agr $^{\mathrm{a}}$ Marilda Torres Capucho; ao laboratorista José Maria Barbosa, pelo auxílio técnico; e ao eng ${ }^{\circ}$ florestal Renato Moraes de Jesus, pela cessão de sementes.

R. Árvore, Viçosa-MG, v.30, n.2, p.171-177, 2006

\section{REFERÊNCIAS BIBLIOGRÁFICAS}

ALVES, M.C.S. et al. Superação de dormência em sementes de Bauhinia monandra Britt. e

Bauhinia ungulata L. - Caesalpinoideae.

Revista Brasileira de Sementes, v.22, n.2, p139-144, 2000.

BEWLEY, J. D.; BLACK, M. Seeds: physiology of development and germination. 2. ed. New York: Plenum Press, 1994. 445p.

BIANCHETTI, A.; RAMOS, A. Comparação de tratamentos para superar a dormência de sementes de canafístula (Peltiphorum dubium). Boletim de Pesquisa Florestal, n.4, p.91-99, 1982.

BRASIL, Ministério da Agricultura e Reforma Agrária. Regras para análise de sementes. Brasília: SNDA/DNDV/CLAV, 1992.365p.

BRUNO, R.L.A. et al. Tratamentos pré-germinativos para superar a dormência de sementes de Mimosa caesalpineaefolia. Revista Brasileira de Sementes, v.23, n.2, p.136-143, 2001. 
CORRÊA, M.P. Dicionário de plantas úteis do Brasil e das exóticas cultivadas. Rio de Janeiro: Imprensa Nacional, 1978. v.6. p.223.

ESCHIAPATI-FERREIRA, M.S.; PEREZ, S.C.J.G.A. Tratamentos para superar a dormência de sementes de Senna macranthera. Revista Brasileira de Sementes, v.19, n.2, p. 231-237. 1997.

JELLER, H.; PEREZ, S.C.J.G.A. Estudo da superação da dormência e da temperatura em sementes de Cassia excelsa Schrad.

Revista Brasileira de Sementes, v.21, n.1, p.32-40, 1999.

LABOURIAU, L.G.; VALADARES, M.E.B. On the germination of seeds Calotropis procera (Ait.) Ait.f. Anais da Academia Brasileira de Ciências, v.48, n.2, p.263-284, 1976.
LOPES, J.C. et al. Germinação de espécies florestais de Caesalpinea ferrea Mart. ex Tul. var. leiostachia Benth., Cassia grandis L. e Samanea saman Merril, após tratamentos para superar a dormência. Revista Brasileira de Sementes, v.20, n.1., p.80-86, 1998.

MAGUIRRE, J.D. Speed of germination-aid in solection and evoluation for seeding emergence and vigor. Crop Science, v.2, n.2, p.176-177, 1962 .

MAYER, A. M.; POLJAKOFF-MAYBER, A. The germination of seeds. 4.ed. Oxford: Pergamon Press, 1989. 270p.

SANTARÉM E.R.; AQUILA M.E.A. Influência de métodos de superação de dormência e do armazenamento na germinação de Senna macranthera. Revista Brasileira de Sementes, v.17, n.2, p.205-209,1995. 